\title{
Industrie néolithique de longues lames en obsidienne, l'exemple d'Aknashen-Khatunarkh (Arménie, début du VIe millénaire) : sur la piste des premiers débitages par pression
}

\author{
Jacques Chabot \\ Université Laval. Département des Sciences Historiques, 1030 avenue des Sciences-Humaines, Pavillon De \\ Koninck, Québec, Qc, G1V 0A6, Canada. Email: jacques.chabot@hst.ulaval.ca
}

\section{Résumé :}

Aknashen (autrefois appelé Khatunakh) est un petit village néolithique de la vallée de l'Ararat localisé à $25 \mathrm{~km}$ de Erevan (capitale de l'Arménie) et à $5 \mathrm{~km}$ au sud-ouest d'Echmiadzin (Vagharshapat). Aknashen est également situé à seulement six kilomètres d'un autre village néolithique qui lui est contemporain : Aratashen, site pour lequel nous avons récemment publié une étude tehnologique du matériel lithique en obsidienne.

Des fouilles ont eu lieu chaque année à Aknashen depuis 2004. En ce qui concerne le matériel lithique, ces travaux ont permis de mettre au jour deux chaînes opératoires principales de matériel en obsidienne (matière très abondante dans cette région) : l'une est peu élaborée sur éclats (expedient tools) et l'autre concerne la confection de longues lames régulières selon plusieurs techniques de débitage. Le présent article portera sur l'industrie laminaire de longues lames régulières obtenues par différentes techniques : pression debout à la béquille, pression au levier et percussion indirecte. La ou les chaînes opératoires qui concernent ces artefacts ne peuvent être reconstituées en entier puisque des éléments manquent et nous ne pouvons pas savoir de façon absolue si par exemple les fines lames débitées par pression à la béquille ont été obtenues après le débitage et la réduction progressive de nucléus d'abord taillés par percussion indirecte, puis par pression au levier. Mais même si divers facteurs nous échappent, dont aussi notamment les lieux exacts où s'est déroulé le débitage, même si la présence d'une panoplie de produits de débitage laisse présumer que le tout se soit déployé en tout ou en partie sur le site, une étude attentive de ce matériel a permis de diagnostiquer les techniques utilisées, mais aussi d'observer le grand niveau de savoir-faire des spécialistes qui ont effectués ce travail de pointe. De façon générale, la pression à la béquille, ainsi que la percussion indirecte, sont les deux techniques d'obtention de longues lames les mieux connues, alors que la pression au levier, même si identifiées dans plusieurs cultures du Néolithique à l'Âge du bronze a fait l'objet de peu d'études de ce genre jusqu'ici. Non seulement ce savoir-faire de haut niveau gagne-t-il à être connu d'un point de vue technologique, mais la reconnaissance du débitage par pression au levier, peut justement constituer un marqueur culturel précieux pour en arriver à caractériser des cultures, des échanges et éventuellement des mouvements ou contacts entre populations.

Dans le cadre de cet article, les principaux spécimens liés à l'identification de chaque technique seront présentés, décrits et commentés et ce matériel sera replacé dans une perspective plus large pour la compréhension de cette culture et de ses origines, autant d'un point de vue de l'histoire des

Published by the School of History, Classics and Archaeology, University of Edinburgh ISSN: 2055-0472. URL: http://journals.ed.ac.uk/lithicstudies/

This work is licensed under a Creative Commons Attribution 2.5 UK: Scotland License. 
techniques et des savoir-faire que pour la connaissance des origines de ce Néolithique encore mal connu. Comme ces recherches sont encore relativement jeunes, bien des aspects sont encore à confirmer et ceci fait partie de travaux qui se poursuivront dans les années à venir.

Mots-clés : technologie lithique; chaîne opératoire; savoir-faire technologique; Neolithique; obsidienne; Caucase; Mésopotamie du nord

\section{Introduction}

Aknashen (autrefois appelé Khatunakh) est un petit village néolithique de la vallée de l'Ararat localisé à $25 \mathrm{~km}$ de Erevan (capitale de l'Arménie) et à $5 \mathrm{~km}$ au sud-ouest d'Echmiadzin (Vagharshapat). Aknashen est également situé à seulement six kilomètres d'un autre village néolithique qui lui est contemporain : Aratashen, site pour lequel nous avons récemment publié une étude technologique du matériel lithique (Chabot \& Pelegrin 2012).

Les fouilles sont encore en cours à Aknashen et font partie du projet " Mission Caucase » dirigé par C. Chataigner (Archéorient, Lyon 2). R Badalyan est en charge des fouilles (Institut d'archéologie et d'ethnographie, Erevan, Arménie) et l'étude du matériel lithique est sous la responsabilité de l'auteur de cet article. Jusqu'ici des campagnes ont été menées de 2004 à 2009, puis de 2011 à 2015. Ces travaux ont permis d'identifier 7 horizons archéologiques : l'horizon I appartient au Chalcolithique (culture Sioni) et les horizons II à V sont datés du Néolithique (culture Aratashen-Shulaveri-Shomutepe). Le niveau VI est une couche d'inondation qui constitue un hiatus entre l'horizon le plus ancien du site (VII) et les autres niveaux. L'horizon VII, identifié en 2013 est daté de 6000 avant l'ère commune (A.E.C.) et fait toujours l'objet de prospections (campagnes 2014, 2015 et 2016).

Il est important de préciser que cette culture néolithique en est une de plain-pied (sans phase préliminaire de développement). Nous sommes donc face à des gens s'étant installés sur ce nouveau territoire avec leur bagage et connaissances, notamment de l'agriculture et de l'élevage, en plus d'une technologie lithique très élaborée qu'ils continueront à déployer tout au long de l'occupation du site, grâce à une dizaine de sources volcaniques d'obsidienne qui nous ont été révélées par des analyses physico-chimiques (Badalyan et al. 2010). Mais ce sont surtout les trois sources, localisées le plus près du village qui ont principalement été utilisées.

Ainsi, 86\% de l'obsidienne a été fourni par: Arteni (48\%) situé à $55 \mathrm{~km}$ au nord-ouest d’Aknashen, Gutansar (32\%) localisé à 50 km au nord-est et Hatis (6\%), volcan localisé au sud de Gutansar et à $45 \mathrm{~km}$ d'Aknashen (Figure 1). Les sources principales du site voisin d'Aratashen étaient les trois mêmes, mais dans des proportions différentes : Arteni (62\%), alors que Gutansar et Hatis comptaient pour moins de 19\% (Badalyan et al. 2007).

Par conséquent, comme ce fut le cas pour Aratashen, une grande quantité de matériel lithique a été mis au jour jusqu'ici à Aknashen, dont l'assemblage contient des milliers d'artefacts entiers, fragmentaires ou lames volontairement segmentées dont une importante partie ont servi en tant qu'outils agricoles (Badalyan et al. 2010; Chabot et al. sous presse).

Or, bien que de telles longues lames régulières sont connues pour des périodes contemporaines et un peu plus anciennes au Proche-Orient voisin (nous y reviendrons plus loin), il n'en demeure pas moins, comme c'était le cas à Aratashen (Chabot \& Pelegrin 2012) que le matériel lithique d'Aknashen, dont les débuts sont datés de 6000 A.E.C., constitue l'une des plus anciennes «industrie » de longues lames attestée à ce jour (aspect systématique du phénomène et non spécimens épars).

Le but de cet article, suite au chapitre sur la technologie d'Aratashen paru en 2012 (Chabot \& Pelegrin 2012), sera précisément de faire une présentation du matériel laminaire et des techniques de débitage de longues lames employées sur ce site voisin: Aknashen qui présente un matériel tout aussi intéressant et une très ancienne industrie de longues lames 
régulières provenant d'une culture néolithique encore méconnue et dont les origines restent à déterminer (l'ensemble du matériel fera l'objet d'une publication complète qui paraîtra dans une monographie en 2018. Ces recherches que nous menons depuis plusieurs années ont toujours été effectuées selon le concept de chaîne opératoire pris dans son sens technologique qui inclut toutes les étapes de la vie d'un artéfact (Inizan et al. 1995) : approvisionnement en matière première, technologie de fabrication des supports, transformation des supports en outils lorsque nécessaire, utilisation, abandon. Le présent article a aussi pour but de replacer ce matériel et ce savoir-faire dans un contexte plus large, dont bien des aspects restent à connaître, car il s’agit malgré tout de recherches relativement jeunes.

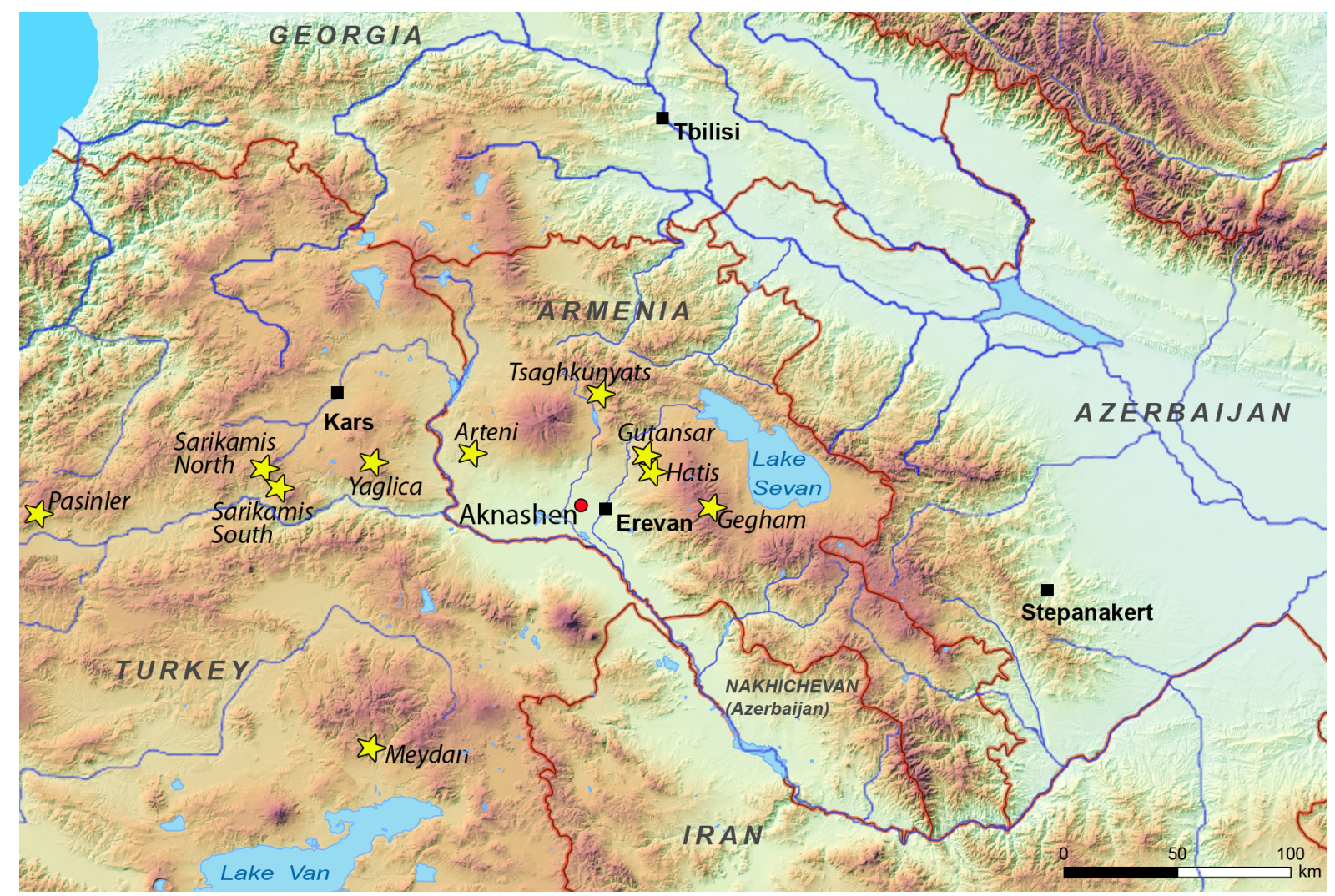

Figure 1. Localisation du village d'Aknashen et des sources d'obsidienne utilisées. (Carte préparée par C. Chataigner, MOM, U. Lyon 2.)

Figure 1. Location of the village of Aknashen and obsidian sources used. (Map prepared by C. Chataigner, MOM, U. Lyon 2.)

\section{Le débitage de longues lames à Aknashen}

Trois techniques ont été identifiées à Aknashen pour le débitage des longues lames régulières: pression debout à la béquille (représentée jusqu’ici par 69 spécimens), pression au levier (48 spécimens) et percussion indirecte (20 spécimens).

Par ailleurs, il peut peut-être paraître surprenant que sur des milliers d'artefacts entiers et fragmentaires, un nombre assez modeste de pièces diagnostiques a pu être associé à une technique. Or, il ne faut pas perdre de vue que ces supports réguliers et de grande qualité étaient destinés à servir (comme l'on démontré nos analyses tracéologiques à fort grossissement : Badalyan et al. 2010; Chabot et al. sous presse - chapitre dans monographie en préparation) et pour se faire, ils ont été modifiés. Ainsi ils sont souvent retrouvés sous forme de petits tronçons (dimensions trop réduites pour diagnostic) qui devaient être emmanchés ou encastrés. Certaines autres pièces ont été cassées et sont découvertes sous forme fragmentaire ne permettant pas non plus de les étudier de ce point de vue. Dans certains 
cas, l'utilisation de certaines pièces (retouches d'utilisation et d'accommodation) a effacé des stigmates importants, empêchant ainsi que l'on puisse diagnostiquer leur technique d'obtention. De plus, il semble que lors de la segmentation volontaire des lames en tronçons, on ait profité de l'occasion pour se débarrasser d'une bonne proportion des parties plus bombées ou courbes (proximales et distales). Le nombre de parties proximales bien conservées demeure donc limité, alors qu'elles sont très importantes pour l'étude technologique de ce type de matériel (comme dans le cadre de toute étude technologique). C'est une situation habituelle sur ces sites (finalité fonctionnelle des artefacts) et il est normal qu'une bonne partie des assemblages soient retrouvés dans cet état. La même situation a été observée pour les longues lames du site d'Aratashen (Chabot \& Pelegrin 2012) et aussi dans des périodes plus récentes sur de nombreux sites du Proche-Orient : phénomène des lames cananéennes (Anderson et al. 2004; Chabot 2015). Par ailleurs, le but de ce type d'analyses n'est pas de toute façon de diagnostiquer toutes les pièces, mais d'en avoir suffisamment pour attester de telle ou telle activité pratiquée par ces populations.

En ce qui concerne les activités de débitage complètes, bien qu'une bonne quantité de produits de débitage a été découverte dans le cadre des fouilles (tous les détails concernant ces produits seront publiés dans la monographie en préparation), ceux-ci ont vraisemblablement fait l'objet d'un nettoyage à partir de leur lieu d'origine (atelier) et sont éparpillés sur le site et jamais concentrés dans un secteur où nous pourrions identifier une aire de taille. Le même phénomène avait aussi été observé à Aratashen (Badalyan et al. 2007). Autre fait important, comme ce fut aussi le cas à Aratashen, certains produits de débitage n’ont pas été retrouvés lors des fouilles. Ainsi nous n'avons pas de longues lames à crête et les grands éclats d'épannelage sont rarissimes. La même tendance est aussi observée à Masis Blur, autre site néolithique contemporain situé à $11 \mathrm{~km}$ d'Aknashen et où les grands éclats de préparation et les crêtes sont aussi très rares (Communication personnelle avec K. Martirosyan-Olshanky en Octobre 2015). Ceci laisse entrevoir qu'une partie du débitage (et donc de la chaîne opératoire) a possiblement été fait ailleurs en dehors du village, ou encore dans un secteur que nous n'aurions pas fouillé encore. De même, nous ne retrouvons pas de grands nucléus desquels auraient été tirés les longues lames. Plusieurs nucléus ont été découverts, mais ils sont courts et surtout associés à la pression à la béquille de plus petite dimension (car cette technique a aussi été pratiquée pour obtenir des lames de modules plus modestes). Jusqu'à un certain point, il est normal de ne pas trouver de grands nucléus (sur le site voisin d'Aratashen quelques-uns avaient été trouvés : Chabot \& Pelegrin 2012), car après une longue préparation méticuleuse, ceux-ci ont forcément été intensément débités. A ce sujet, il n’est pas impossible que ces petits nucléus d'une dizaine de $\mathrm{cm}$ et moins que nous trouvons, sont peut-être des nucléus à longues lames qui ont été peu à peu réduits et recyclés pour en tirer des supports plus petits. Quelques-uns ont été publiés (Badalyan et al. 2010) et plusieurs autres feront partie de la synthèse que nous préparons actuellement.

Par ailleurs, comme l'industrie laminaire d'Aknashen, est homogène d'un point de vue technologique d'un horizon à l'autre (pas de différence notoire dans l'utilisation des techniques tout au long de l'occupation du site), le matériel sera traité globalement dans le cadre de cet article et illustré avec les meilleurs exemples. Mentionnons que ce site comporte aussi une chaîne opératoire sur éclats (débitage ad hoc peu élaboré) qui sera publiée dans la synthèse que nous préparons actuellement.

\subsection{Le débitage par pression debout à la béquille}

Les artefacts diagnostics liés à la pression debout à la béquille à Aknashen sont pour le moment (fouilles en cours) au nombre de soixante-neuf. Il s'agit de pièces très bien conservées et qui présentent des caractéristiques claires. Nous en reproduisons ici quelques 
exemples (Figure 2 : a, b, c, d, e, et f). Bien sûr, de nombreux autres artefacts découverts sur le site ont certainement été obtenus grâce à cette technique, mais ils sont conservés dans un moins bon état.

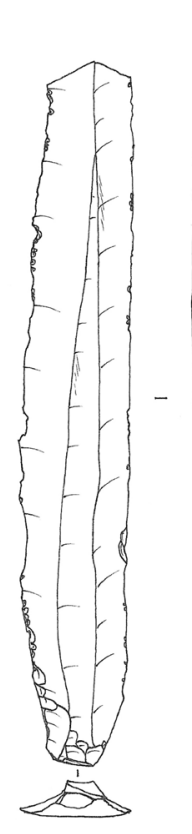

a
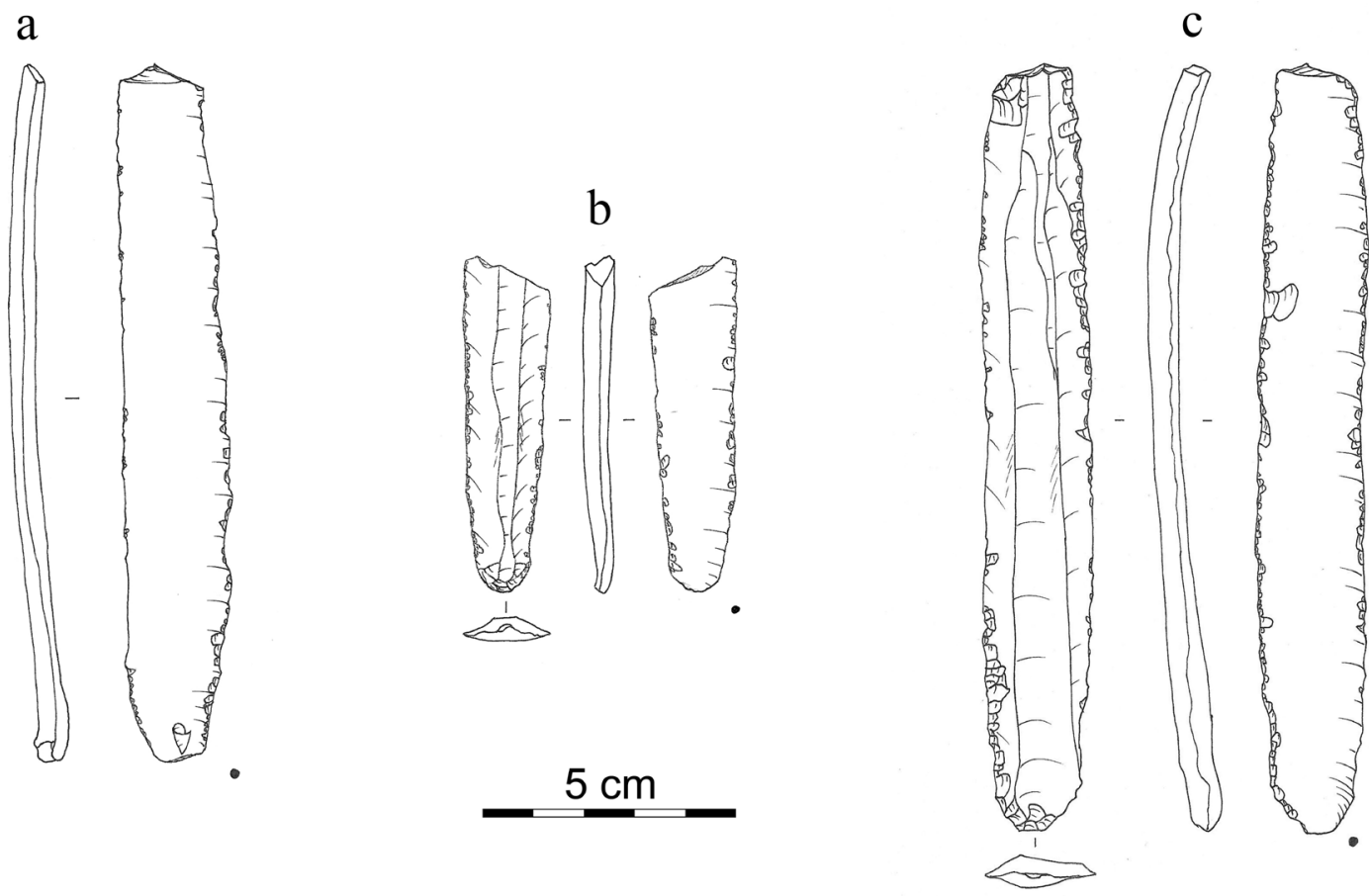

d
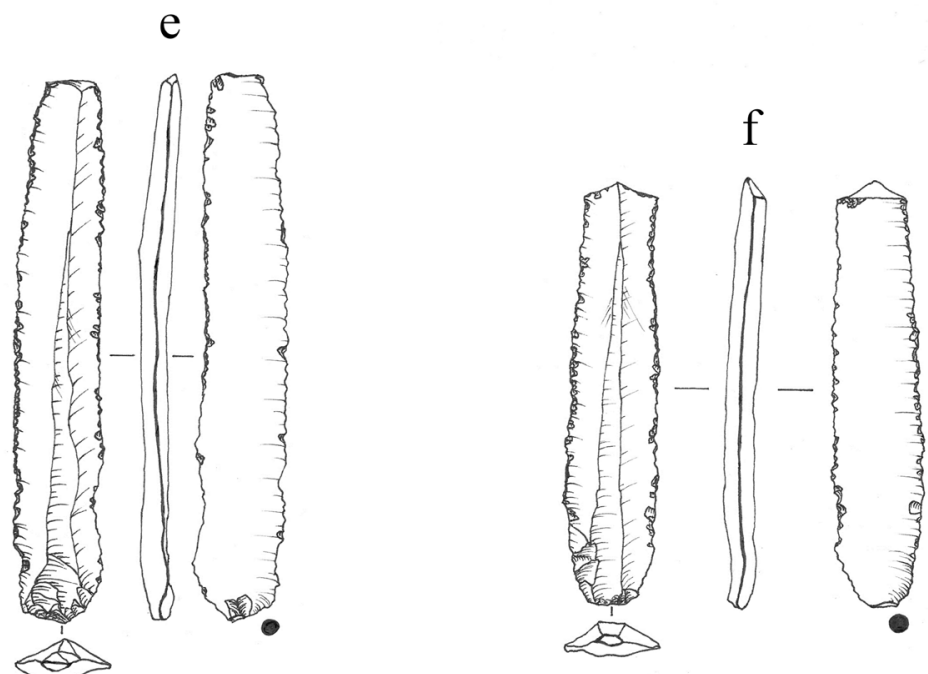

Figure 2. Tronçons de lames d’Aknashen fabriqués par pression debout à la béquille.

Figure 2. Segments of blades from Aknashen knapped by standing up pressure with a crutch.

Ultimement, en ce qui concerne le débitage par pression, le diagnostic peut être posé par la présence de trois critères sur une même pièce, soit: grande régularité des bords et des nervures, rectitude du profil et section légère (Tixier 1984, Pelegrin 2002 - dans Chabot 2002; Pelegrin 2012b). En ce qui concerne nos travaux récents sur du matériel archéologique, nous avons pu vérifier la présence de ces trois critères réunis sur du matériel de plusieurs sites du Proche-Orient et du Caucase (Anderson et al. 2004, Chabot 2002, Chabot 2015, Chabot \& Pelegrin 2012 pour n'en nommer que quelques-uns).

Morphologiquement, l'aspect dominant chez ces lames et tronçons de lames fabriqués par pression debout à la béquille est leur finesse et leur légèreté (aspect élancé et beaucoup moins robuste que les autres longues lames). Pour des raisons fonctionnelles, comme nous le 
mentionnons ailleurs dans ce texte, elles sont rarement retrouvées entières car leur partie distale courbée était une nuisance d'un point de vue fonctionnel. Leur longueur oscille ainsi de 4,4 à $15 \mathrm{~cm}$ pour les éléments qui ont conservé leur partie proximale. La lame la plus large rattachée ici à cette technique mesure $22,7 \mathrm{~mm}$, mais la moyenne de l'ensemble de ces lames est de 15,3 mm.

A ce propos, le débitage par pression debout à la béquille sur obsidienne peut permettre d'extraire des lames pouvant atteindre jusqu'à $28 \mathrm{~mm}$ de large. Ceci a notamment été démontré par les recherches expérimentales de J. Pelegrin (Communication personnelle avec J. Pelegrin en juillet 2015).

Une quarantaine d'autres tronçons de lames d'Aknashen ont entre 24 et $28 \mathrm{~mm}$ de large, mais on ne peut discriminer précisément si elles ont été débitées à la béquille ou par pression au levier, car ces spécimens présentent trop de caractères qui pourraient appartenir à l'une ou l'autre technique. Ce sont des pièces qui sont classées dans une catégorie «débitage par pression ", mais pour lesquelles nous ne pouvons préciser davantage. Finalement, les lames diagnostics de pression à la béquille d'Aknashen ont une épaisseur moyenne de 3,7 mm.

Les caractères techniques propres à la pression à la béquille se remarquent tout d'abord dans la partie proximale : surface de contact assez réduite (talon punctiforme, linéaire ou petit et lisse) et petit bulbe saillant haut et court. De tels petits talons sont attribuables à une pression effectuée le plus près possible du bord du nucléus afin d'économiser la force et de faciliter le détachement de la lame. L'extrémité proximale est plus étroite que le corps de la lame. Les pièces présentent un profil rectiligne, une minceur relative, une face d'éclatement lisse (témoin de la stabilité du système de débitage) et une courbure qui ne s'amorce qu'en distal. Au niveau de la face supérieure, on remarque une grande rectitude et un parallélisme des bords et des nervures.

La technique par pression que ce soit par béquille ou au levier, offre cet avantage éventuel d'un point de vue fonctionnel à savoir: l'obtention de longues lames régulières qui permettent d'obtenir plus de tronçons par lame. Dans le cadre de ce contexte néolithique, ceci n’est pas à négliger puisqu'une grande proportion de ces produits était destinée à servir dans des outils agricoles composites (notamment dans des faucilles et tribulums comme nos recherches l'ont démontré).

\subsection{Le débitage par pression au levier}

A Aknashen jusqu'ici, quarante-huit artefacts ont pu être diagnostiqués comme ayant été fabriqués à la pression au levier. Il s’agit de vingt-deux tronçons proximaux-mésiaux (PM), dix-neuf tronçons mésiaux $(\mathrm{M})$, quatre tronçon mésiaux-distaux et trois lames entières d'environ $15 \mathrm{~cm}$, longueur plutôt modeste pour ce type de technique. Il n'est guère surprenant de constater que les tronçons proximaux-mésiaux et mésiaux dominent l'assemblage. En effet la partie mésiale de ces lames, offraient un profil très rectiligne (optimum d'un point de vue fonctionnel) et la partie proximale, quant à elle, même si un peu en relief, présente un bulbe assez discret qui ne devait pas être une entrave fonctionnelle, comparativement à d'autres technique comme la percussion indirecte par exemple. Avec cette technique de pression, la stabilité est rapidement atteinte et le début de la partie mésiale situé juste après l'extinction du bulbe, est très rectiligne et lisse.

Les segments de lames identifiés à cette technique ont une largeur entre 24,5mm et 34,7 mm (largeur moyenne de $30 \mathrm{~mm}$ : une telle largeur étant impossible à atteindre par pression debout à la béquille : Chabot \& Pelegrin 2012) et une épaisseur moyenne de 5,4 mm (voir exemples représentés ici : Figure $3:$ a, b, c, d, e, f, et g). 


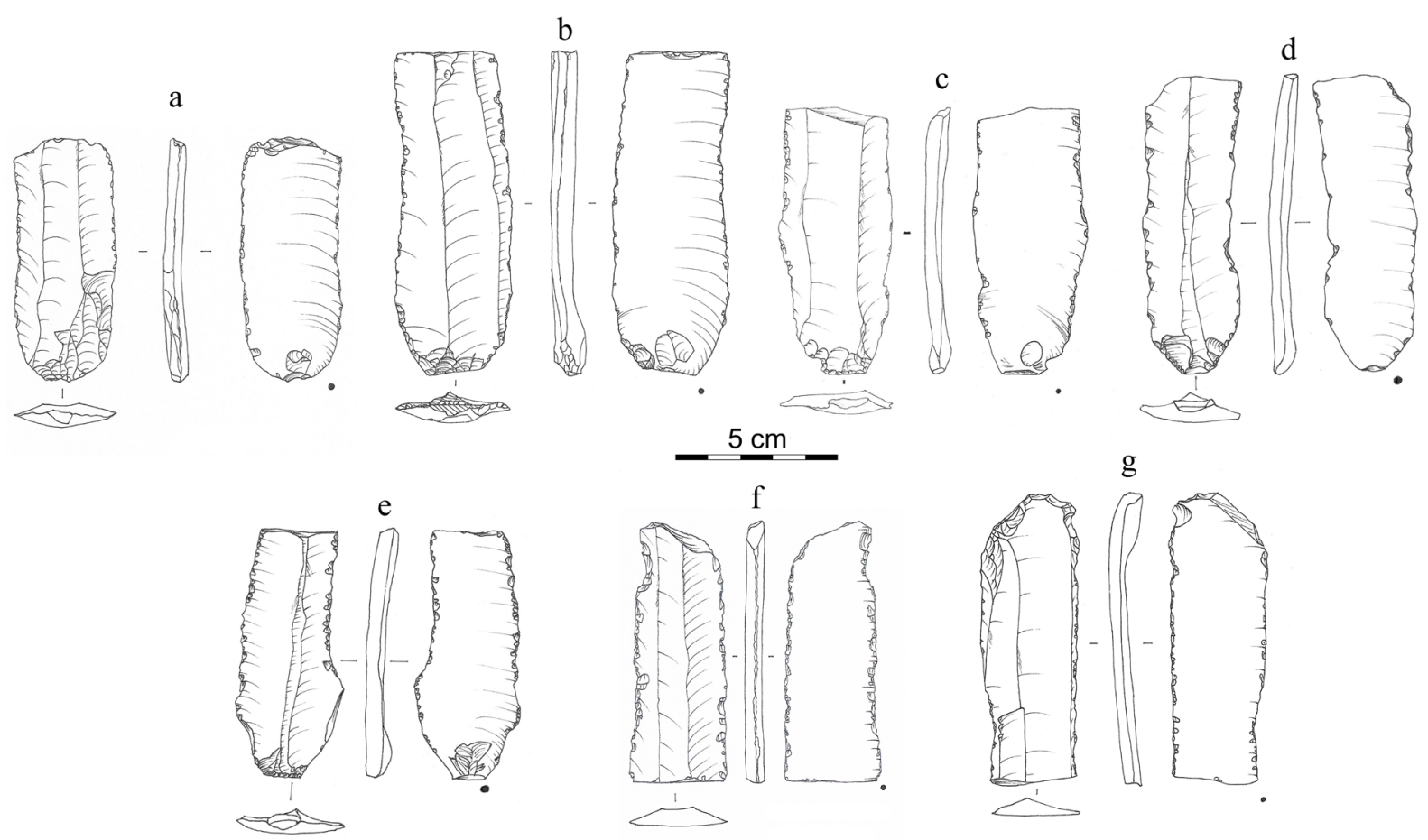

Figure 3. Tronçons de lames d'Aknashen fabriqués par pression au levier.

Figure 3. Segments of blades from Aknashen knapped by pressure with a lever.

Dans plusieurs publications, la méthode de fabrication de lames par pression au levier a été décrite (Anderson et al. 2004; Chabot \& Pelegrin 2012; Pelegrin 2012b; Pelegrin 2002 dans Chabot 2002). Pour en arriver à l'étape finale du détachement des lames par pression au levier, dans le cadre de cette méthode, il faut préalablement effectuer le dégrossissage du bloc par percussion directe au percuteur dur de pierre, puis la régularisation du nucléus est effectuée par percussion indirecte et une fois cette étape accomplie, le nucléus est prêt pour le débitage par pression au levier qui s'effectuera dans un système fixe, tel un arbre évidé. La pointe dont sera muni le levier peut être faite d'une pièce de bois armée d'une pointe de cuivre ou d'un andouiller de cervidé (Figure 4) (voir également Pelegrin 2012b).

L'utilisation de telle ou telle pointe peut être détectée sur le talon ou à la jonction du talon et de la face d'éclatement, Ainsi, sur de telles pièces, un talon fissuré, est révélateur de l'utilisation d'une pointe métallique, alors qu'une pointe en bois de cervidé détachera plutôt par arrachement et verra la formation d'une petite lèvre (Chabot 2002; Chabot \& Pelegrin 2012; Pelegrin 2002 - dans Chabot 2002; Pelegrin 2012a; 2012b; ).

Ainsi la pièce de la Figure 3d présente une petite lèvre et une absence de fissure sur le talon ce qui dénote fort probablement l'emploi d'une pointe en bois de cervidé. Les pièces représentées sur les Figures $3 b$ et $3 e$ ont une discrète fissuration du talon, preuve probable de l'utilisation d'une pointe métallique (fort probablement en cuivre) pour les détacher du nucléus par pression au levier. De tels stigmates ont pu être observés sur quelques pièces de l'assemblage, alors que dans certains cas, il n'est pas possible de détecter la nature de la pointe. Nous avions déjà détecté de telles caractéristiques sur le matériel d'Aratashen (Chabot \& Pelegrin 2012) ainsi que sur des lames cananéennes du Proche-Orient (Chabot 2002) et ce sont les expérimentations de J. Pelegrin qui ont permis de mettre en lumières la présence de paramètres aussi fins. 


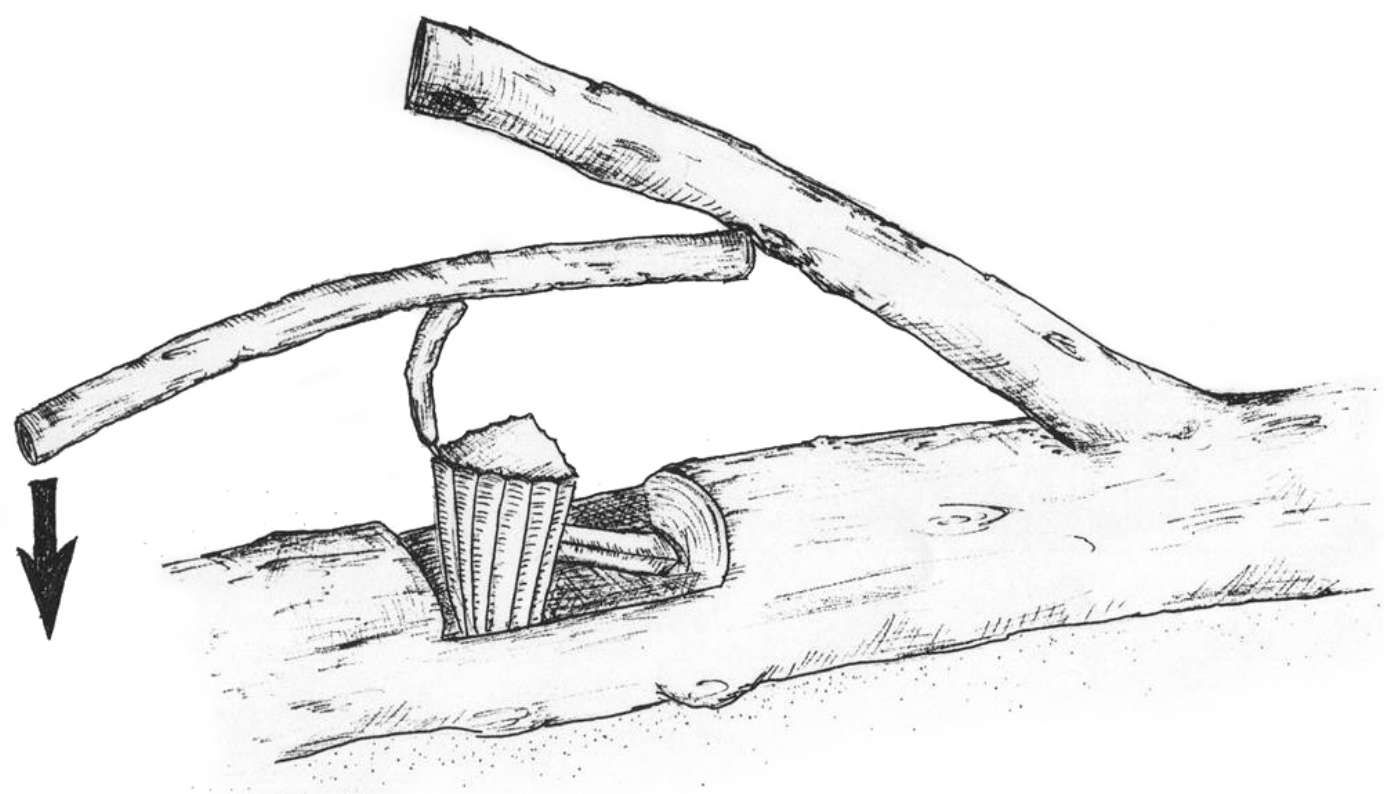

Figure 4. Système expérimental de débitage par pression au levier.

Figure 4. Experimental system of pressure knapping with a lever.

Par ailleurs, sur les tronçons de lames obtenus par pression au levier, on détecte les mêmes trois critères de régularité que nous avons décrits pour la pression debout à la béquille : régularité des bords et des nervures, rectitude du profil et légèreté de la section. Ainsi, la pression au levier est une technique qui permet en démultipliant la force (pression sur le nucléus pouvant aller jusqu'à $300 \mathrm{~kg}$ ), d'obtenir des lames plus longues et plus larges, mais les critères de régularité de la pression y demeurent bien présents. D’autres caractères typiques de la pression sont aussi observables, comme : bulbe saillant haut et court, face d'éclatement qui devient lisse et plate juste après l'extinction du bulbe, courbure en distal uniquement.

Aussi, dans ces assemblages, les talons lorsque conservés, sont toujours lisses, mais de dimensions plus grandes que pour les lames obtenues par pression à la béquille. L'extrémité proximale demeure néanmoins moins large que le corps de la lame. On peut aussi observer sur certaines pièces que la corniche a été abrasée. La grande régularité des bords et des nervures peut également être observée sur le tronçon mésial et mésial-distal (légèrement courbé) (Figure $3: \mathrm{f}$ et g).

Par conséquent, sur bien des aspects, les lames et éléments de lames fabriqués par pression au levier peuvent beaucoup ressembler à ceux fabriqués par pression à la béquille. Mais comme nous venons de le voir, la pression au levier permet d'aller plus loin techniquement, dans le sens où elle offre des produits tout aussi réguliers et rectilignes, mais sur de plus gros modules et donc des lames plus robustes. En ce sens, elles conservent cet avantage qu'offrait la percussion indirecte (que nous allons observer plus loin), mais avec une grande régularité dans les proportions que seule une technique par pression permet d'obtenir pour des lames de cette dimension.

Toujours au sujet de ces ressemblances entre pression à la béquille et pression au levier, comme nous l'avons souligné plus haut, à Aknashen nous avons identifié quarante spécimens de lames régulières que l'on sait avoir été débitées par pression, mais pour lesquelles on ne peut trancher pour une ou l'autre technique, vu que leur largeur occupe un spectre qui peut être obtenu autant par la pression à la béquille (limite de cette technique) que par celle au 
levier (soit entre 24 et $28 \mathrm{~mm}$ de large) et dont le talon, relativement petit sur ce échantillonnage, ne permet pas non plus de discriminer pour une technique ou l'autre.

Le fait d'utiliser des techniques de débitage par pression aussi complexes n'est pas anecdotique. En effet, en ce qui concerne les qualités intrinsèques de l'obsidienne en tant que matière première (verre volcanique), il n'aurait pas été possible de fabriquer des objets de cette dimension, aussi réguliers, minces et standardisés par d'autres techniques, comme la percussion directe (Chabot \& Pelegrin 2012).

\subsection{Débitage par percussion indirecte}

Vingt artefacts provenant d'Aknashen ont jusqu'ici pu être identifiés à la percussion indirecte. La largeur moyenne de ces pièces est de $31 \mathrm{~mm}$ et leur épaisseur moyenne est de 6 mm. Presque tous les tronçons sont des segments proximaux-mésiaux, un est mésial et un est mésial-distal. Parmi les pièces associées à cette technique, certaines sont des tronçons assez courts, mais qui présentent assez de caractères nets pour effectuer ce classement. Encore une fois, même si la percussion indirecte a possiblement joué un rôle plus discret, utilisée pour la mise en forme des nucléus et peut-être aussi pour leur réaménagement en fin de parcours, dans la plupart des cas les pièces ainsi détachées, étaient de bons outils potentiels et il n'est guère surprenant de les retrouver ainsi volontairement fragmentés, comme c'est aussi le cas pour les autres types de longues lames d'Aknashen. Il est donc normal et peu surprenant de retrouver peu de lames indemnes. Le même phénomène a aussi été observé sur le matériel d'Aratashen (Chabot \& Pelegrin 2012) et de nombreux sites du Proche-Orient sur lesquels nous avons travaillé (voir notamment: Chabot 2002; Chabot 2015; Chabot \& Eid 2007; 2010).

Plusieurs caractéristiques font en sorte que ces lames se distinguent des pièces obtenues par pression (Figure 5 : a, b, c d). Tout d'abord, elles présentent un talon lisse souvent assez épais, mais presque toujours très long faisant en sorte que la partie proximale est presque toujours aussi large que le corps de la lame; les talons de ces types de lames ont au moins 0,8 mm et plus de large et une profondeur d'au moins 3 ou 4 mm (Pelegrin 2012 b).

Ceci peut d'ailleurs donner un aspect carré à l'extrémité proximale. Le bulbe est très saillant, large et étendu. La lame présente souvent une courbure marquée qui s'amorce dès la partie mésiale donnant souvent un aspect globalement arqué à la lame. Des bombements sont souvent présents sur la face d'éclatement, preuve que ces pièces proviennent d'un système de débitage moins stable. Les bords et nervures sont souvent plutôt réguliers mais pas aussi droites que celles obtenues par pression, même si la chose est possible surtout chez certains spécimens sur plus petit module ou encore qui ont été conservés sur courte longueur (tronçons). La section est souvent lourde (large et épaisse) et les produits sont donc plus robustes d'aspect, comme le montrent les épaisseurs des lames. Ainsi, même si ces produits peuvent être assez réguliers en général, on ne retrouvera jamais réuni sur une même, les trois critères de régularité mentionné pour le débitage par pression (Chabot 2002; Chabot \& Pelegrin 2012).

D’un point de vue fonctionnel, la courbure marquée dès la partie mésiale, observée chez ce type de lame, ne permet pas de façon générale, d’obtenir beaucoup de tronçons plats en les segmentant (contrairement aux pièces obtenues par pression au levier). Ceci est peut-être une partie de l'explication à savoir pourquoi la percussion indirecte semble avoir joué un rôle moins important. 

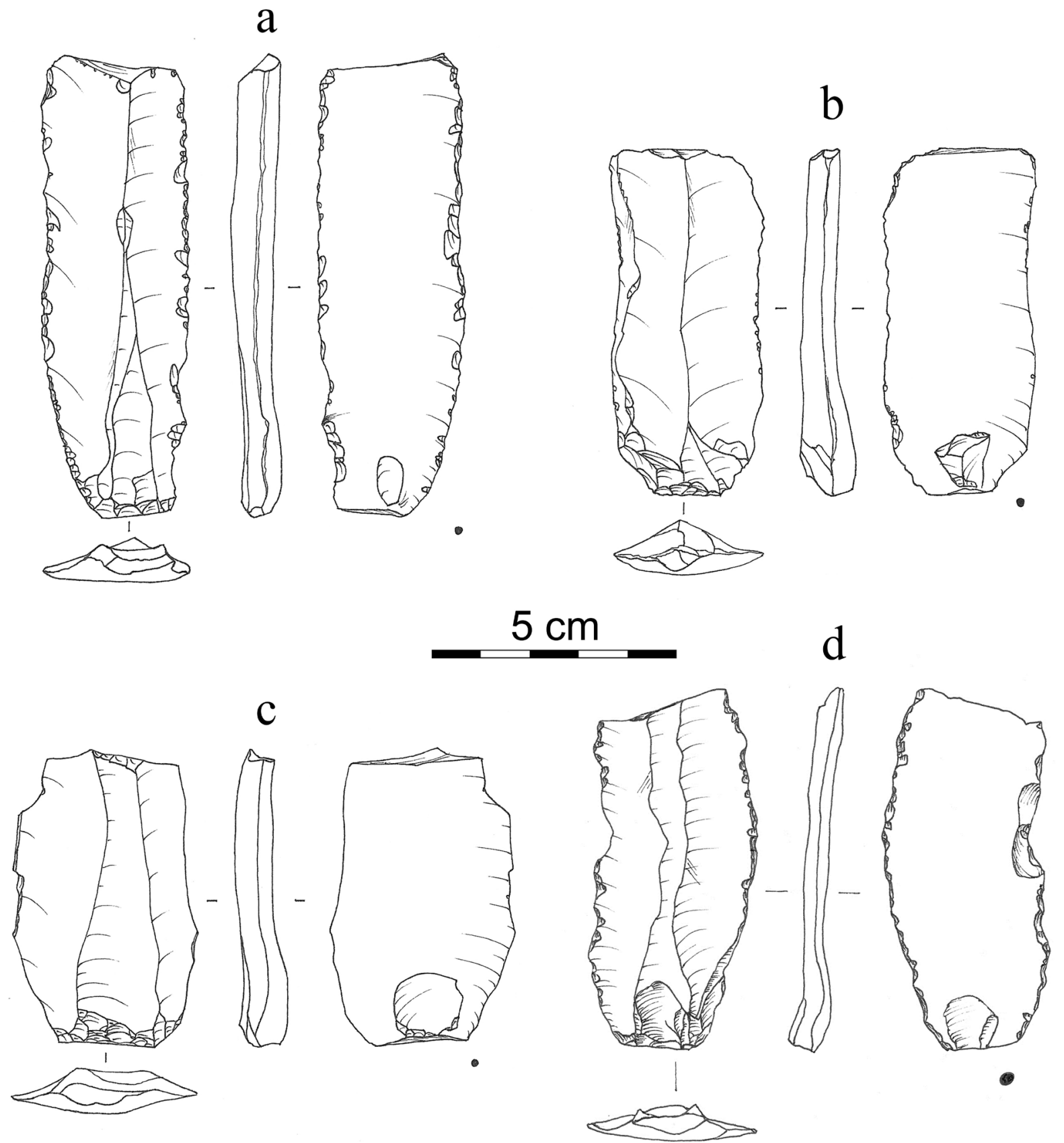

Figure 5. Tronçons de lames d’Aknashen fabriqués par percussion indirecte.

Figure 5. Segments of blades from Aknashen knapped by indirect percussion.

\section{Pourquoi de la pression au levier?}

Théoriquement, le fait de fabriquer des lames par pression au levier n'était pas essentiel (Chabot \& Pelegrin 2012), car, comme nous venons de le voir, il était possible d'obtenir des lames régulières par pression à la béquille et assez régulières et robustes aussi par percussion indirecte. Pourquoi alors se donner autant de mal pour fabriquer ces artefacts? Ceci nous ramène une fois de plus au contexte du Néolithique, Chalcolithique et de l'Âge du Bronze Ancien, périodes où de telles lames sont très présentes en Mésopotamie septentrionale et au Caucase du sud notamment. L'invention et le développement progressif de l'agriculture qui petit à petit s'étendra à un vaste territoire en devenant la base et le principal moyen de subsistance de ces sociétés, en est peut-être la cause. Ainsi les activités de récoltes (moisson) et le traitement de celles-ci par dépiquage au tribulum (fonctions identifiées par analyses 
tracéologiques- voir Badalyan et al. 2010; Chabot et al. sous presse) nécessitaient un excellent outillage standardisé afin de concevoir des traîneaux à dépiquer équilibrés. Ceux-ci étaient essentiellement conçu par des rondins liés grâce à des liens en cuir et donc les éléments tranchants devaient être encastrés entre les rondins et être standards en termes de dimensions pour que le traîneau soit équilibré. Ceci a notamment été démontré grâce aux expérimentations menées par P. Anderson (CNRS, Nice). A ce sujet, pour en arriver à documenter ce matériel d'un point de vue fonctionnel nous avons participé à plusieurs de ces programmes expérimentaux sur longues lames et outils agricoles menés en Provence depuis longtemps (notamment en 1997, de 1999 à 2001 et en 2011). De plus, nous avons dirigé un important programme expérimental qui s'est déroulé à notre laboratoire de Québec (université Laval) en 2013 et 2014, concernant les outils qui ont connu une utilisation de courte durée. Ces expérimentations concernaient spécifiquement les outils ad hoc sur éclats d'Amérique du nord (Chabot et al. 2014; 2017), mais nous en avons profité pour inclure dans ce projet l'obsidienne d'Arménie afin d'enrichir nos référentiels et de mieux comprendre ce matériel.

Ainsi, il n'est guère surprenant de constater qu'un lien aussi étroit existe entre technologie et finalité fonctionnelle (deux étapes fondamentale de la chaîne opératoire d'un artefact). Ce sont des aspects que nous avons maintes fois abordés et qui dépassent toutefois les objectifs du présent article (voir par exemple : Anderson et al. 2004; Chabot 2002; 2015; Chabot \& Eid 2007).

De plus, de telles lames obtenues par pression, offrent aussi un autre avantage lié à la fonction (comme mentionné plus haut), à savoir la possibilité d'extraire plusieurs tronçons rectilignes par lame, vu que seule leur partie distale était courbée. Donc la pression permettait d’obtenir des lames aux proportions régulières (prévisibles) et une plus grande partie de chaque lame pouvait être utilisée. La pression au levier quant à elle, offre des produits tout aussi réguliers que la pression à la béquille, mais des pièces plus robustes. Il existe peut-être d'autres raisons à la présence de ces types de débitage, mais ceci est possiblement un début d'explication. Ainsi la pression au levier offre un double avantage, en ce sens, elle possède la grande régularité que l'on retrouve chez la pression à la béquille, mais tout en offrant en plus une certaine robustesse des produits que la percussion indirecte possédait (sans en avoir la régularité).

Toujours d'un point de vue fonctionnel, il faut aussi noter que cette vocation agricole pour une majorité de ces produits laminaires, explique probablement aussi pourquoi ces artefacts portent si peu de retouches intentionnelles du bord. Ces dernières n'étant pas nécessaires pour bien couper et hacher les plantes (un tranchant brut demeure le plus efficace pour ces tâches).

\section{Origines de ces débitages par pression}

Par ailleurs, un aspect important concernant ce débitage de longues lames du Néolithique caucasien, est son origine. En effet, puisque les premiers néolithiques du Caucase arrivèrent sur ce territoire vers 6000 A.E.C., avec leur bagage déjà constitué, ceci signifie que leur connaissance de ce débitage par pression remonte forcément à des périodes plus anciennes. Il s'agit d'un sujet sur lequel davantage de recherches devront être faites dans les années à venir, afin de connaître précisément l'origine et les routes empruntées par ces techniques complexes de débitage par pression, mais nous pouvons dégager quelques éléments basés sur d'autres recherches technologiques récentes effectuées sur les territoires de Mésopotamie septentrionale et d'Anatolie (Altmbilek et al. 2012; Astruc 2007; Binder 2007; Pelegrin 2012b) qui semblent être probablement les seuls endroits connus pour le moment ayant recelé des lames d'obsidienne comparables et plus anciennes. 
Ainsi, en ce qui concerne ces cultures voisines et situées à l'ouest du Caucase, bien que la pression à la béquille est attestée depuis la $2^{\mathrm{e}}$ moitié du IXe millénaire (Pelegrin 2012b) et a été identifiée sur plusieurs sites de Syrie et de Turquie (Altmbilek et al. 2012), ces plus anciens exemplaires concernaient des lamelles obtenues par pression assise à la mini-béquille (Pelegrin 2012b) et ce n'est qu'à partir de 7340 A.E.C. que des spécimens de longues lames en obsidienne obtenues par pression seront identifiés à Cayönü. (Altmbilek et al. 2012). Parmi l'assemblage, une lame presque complète, et quelques éléments fragmentaires de lames ont pu être associées à la pression au levier (Altmbilel et al. 2012). D’autres pièces semblables ont aussi été découvertes à Sabi Abyad en Syrie du nord dans des niveaux datés de 6500 A.E.C. (Altmbilek et al. 2012). Ces dernières ont été fabriquées sur obsidienne exogène (Bingöl) et comme pour les artefacts de Cayönü, on ne connaît pas exactement de quel atelier elles proviennent. A Sabi Abyad, dix fragments de lames ont pu être associés à la pression au levier. A Cayönü comme à Sabi Abyad, l'examen des talons conservés ne montre aucune fissuration, ce qui tend à démontrer que de la pression à pointe en bois de cervidé était pratiquée, alors qu'à Aknashen (comme aussi sur le site d'Aratashen), les deux types de pointes ont probablement servi (andouiller et cuivre).

Une différence importante entre ces industries et celles du sud Caucase réside (pour le moment) dans les effectifs identifiés et dans l'origine du débitage lui-même. En effet on ne sait pas exactement où ont été débitées les industries du Proche-Orient; elles sont exogènes autant à Cayönü qu’à Sabi Abtad (Altmbilek et al. 2012) et plus de travaux seront nécessaires pour arriver à mieux caractériser l'origine de ces pièces.

A Aknashen, même si la localisation précise des ateliers de taille reste à faire, tout semble indiquer que ce débitage s’est néanmoins déroulé près du site (ou en partie sur le site) ou à tout le moins, non loin des sources principales identifiées autour du site et d'où la grande majorité de l'obsidienne provient. Ceci explique les effectifs nettement plus nombreux et l'aspect systématique de cette industrie. Ainsi comme nous l'avons vu, 157 pièces ont pu être associées à du débitage par pression, dont 48 à la pression au levier et 40 autres pièces qui ont aussi été débitées par pression (au levier ou à la béquille) et les recherches qui se poursuivent feront augmenter ces effectifs. L'abondance du matériel, place Aknashen parmi les plus anciens témoins d'une industrie sur longues lames standardisées (ce dès 6000 A.E.C.).

Par ailleurs, le haut degré de connaissances techniques nécessaire pour produire un tel débitage si élaboré a pu être mesuré grâce aux nombreuses expérimentations menées par J. Pelegrin depuis plusieurs années. (Pelegrin 1988, Pelegrin 2012a). Encore une fois, il est intéressant d'observer que les premiers néolithiques du Caucase possédaient déjà cet avancement technologique dès leur implantation sur ce territoire.

Comme nous l'avons déjà mentionné (Chabot \& Pelegrin 2012), il est très envisageable que l'origine du débitage par pression du Caucase du sud soit à chercher en Anatolie, chez des communautés de Bingöl et de Nemrut Dag localisées à $250 \mathrm{~km}$ au sud-ouest de l'Araxe et qui taillaient l'obsidienne par pression, mais nous en savons peu sur la perduration de cette technique en ce secteur et surtout si la pression au levier y était encore pratiquée vers 6000 A.E.C.

\section{Origines de cette culture et finalité de ce débitage}

Le Néolithique du sud du Caucase est une région riche en obsidienne, mais encore peu connue pour ce qui est du Mésolithique et du Néolithique. Par conséquent, l’origine du Néolithique arménien reste à déterminer. Il s'agit d'un Néolithique complet et pleinement établi (agriculture et élevage) dès le début de l'occupation du site (6000 A.E.C.). Traditionnellement il était considéré que des gens du Proche-Orient seraient possiblement venus au Caucase pour y exploiter les sources d'obsidienne. Mais, cette hypothèse s'est 
révélée fausse, car dans les faits, ces objets en obsidienne confectionnés selon un grand savoir-faire technologique, ne semblent pas avoir circulé hors du Caucase. En effet les analyses physico-chimiques menées sur les divers sites du Proche-Orient et du Caucase, ont révélé que ces dernières sont absentes des sites localisés hors du sud Caucase et donc, elles n’ont pratiquement pas circulé (Badalyan et al. 2007; Badalyan et al. 2010). D’autant plus que d'un point de vue archéologique, si ces artéfacts remarquables avaient été présents sur des sites localisés hors du Caucase, ce n'est pas nécessairement avec nos recherches qu'ils auraient été révélés, car ce matériel très original aurait été signalé depuis longtemps, ce qui ne fut pas le cas.

Nous l'avons évoqué plus haut, Aknashen a fourni une certaine quantité de produits diagnostiques, à travers les milliers d'objets lithiques découverts. Or, on est en droit de se demander pourquoi ce site, comme les villages voisins (même phénomène notamment à Aratashen), qui somme toute sont de modestes villages, renfermaient autant de matériel lithique, surtout, comme nous venons de l'évoquer que ce matériel localement fabriqué ne semble pas avoir été voué à l'exportation.

\section{Conclusion}

Pour revenir à l'origine de cette culture qui comprend des influences multiples entre Proche-Orient et Caucase du nord, la poursuite de l'étude technologique de ce matériel, pourrait constituer un élément clé déterminant pour préciser ses origines. En effet, le haut degré d'avancement technologique nécessaire pour effectuer ces débitages par pression peut constituer un marqueur culturel important (Inizan 1991), d'où l'importance d'identifier et de comprendre ces innovations technologiques, datables via les niveaux archéologiques d'où ils proviennent et de reconstituer les routes qu'elles ont empruntées (transferts d'idées et de technologie). Ainsi, dans le cas présent, la pression au levier, pourra peut-être constituer un élément majeur afin d'éventuellement permettre de distinguer d'où exactement provenaient ces gens. Par contre, comme c'est souvent le cas au Néolithique, le brassage d'idée, le développement et les emprunts de diverses techniques font en sorte que les cultures observées dans des néolithiques établis (sans phase de développement, comme c'est le cas d'Aknashen), présentent bien souvent un aspect hybride dont l'origine ou les origines diverses demeurent très complexes à retracer (Cauvin 1989). En archéologie, c'est souvent dans les détails que se trouvent les réponses. De ce fait, l'étude de ces technologies de pointe, œuvre de spécialistes, dans une société néolithique qui ne cessera elle-même de se spécialiser, demeure certainement une clé très importante pour arriver à mieux comprendre ces cultures et leurs interactions.

\section{Remerciements}

Ministère des Affaires Étrangères et du développement international (M.A.E.D.I., France) qui finance la Mission Caucase

Équipe de recherche en archéométrie de l’Université Laval (CELAT) pour leur appui constant (financier et scientifique) concernant nos travaux en Arménie.

Dr Christine Chataigner, responsable de la mission Caucase (Maison de l'Orient Méditerranéen, Lyon 2, UMR 5133 Archéorient), son aide et son expertise scientifique ont toujours été d'une importance capitale pour la réalisation de nos travaux et de toute cette mission.

Dr Ruben Badalyan, Institut d'archéologie et d'ethnographie, Erevan, pour son accueil dans son beau pays et pour avoir toujours généreusement partagé ses connaissances avec nous à propos de cette culture et de l'archéologie de cette région fascinante.

Dr Jacques Pelegrin, Laboratoire « Préhistoire et technologie », CNRS et Université Paris Ouest Nanterre. Nos échanges fréquents depuis tant d’années sur la complexe question des 
débitages de longues lames et les spécimens découverts, sont très précieux dans le cadre de nos travaux.

Dr Patrick Eid (Université de Montréal), Cynthia Gosselin (Université Laval) et Lorenzo Alberton qui ont été des assistants et des interlocuteurs exceptionnels durant plusieurs missions en Arménie.

\section{Références}

Altmbilek-Algül, C., Astruc, L., Binder, D \& Pelegrin J. 2012, Pressure Blade Production with a Lever in the Early and Late Neolithic of the Near East. In: The Emergence of Pressure Blade Making. From Origins to Modern Experimentation (Desrosiers, P., Ed.), Springer, New York: p. 157-179. (en anglais) (« Production de lames par pression au levier au Néolithique ancien et récent au Proche-Orient »)

Anderson, P. \& Aigouy, P. (directors) 2001, Outils, techniques et tablettes. Sur les traces de savoirs agricoles d'autrefois. Centre interuniversitaire d'Études sur les Lettres, les Arts et les Traditions (CELAT), Université Laval, Québec. film-vidéo. Running time: 25 minutes. (in French) (“Tools, Techniques and Tablets")

Anderson, P., Chabot, J., Van Gijn. A. 2004, The Functional Riddle of 'Glossy’Canaanean Blades: The Apogee of the Mesopotamian Threshing Sledge. Journal of Mediterranean Archaeology, 17(1): 87-132. (en anglais) («L’énigme fonctionnelle des lames cananéennes lustrées ») doi:10.1558/jmea.17.1.87.56076

Astruc, L., Gratuze, B., Pelegrin, J.\& P. Akkermans. 2007, From Production to use: a Parcel of Obsidian Bladelets at Sabi Abyad II. In: Systèmes techniques et communautés du Néolithique précéramique (Astruc, L., Binder, D., \& Briois, F. Eds.), Éditions A.P.D.C.A., Antibes: p. 327-341. (en anglais) (« De la production à l’utilisation : un lot de lamelles en obsidienne de Sabi Abyad II »)

Badalyan, R., Harutyunyan, A., Chataigner, C., Le Mort, F., Chabot, J., Brochier, J-E., Balasescu, A., Radu, V\& Hovsepyan, R. 2010, The Settlement of AknashenKhatunarkh, A Neolithic Site in the Ararat Plain (Armenia) (excavation results 20042009), TÜBA-AR Turkish Academy of Sciences Journal of Archaeology, 13:185-218. (en anglais) ( « L’établissement d'Aknashen, un site de la vallée de l'Ararat (résultats des fouilles de 2004-2009»)

Badalyan, R., Lombard, P., Avetisyan, P., Chataigner, C., Chabot, J., Vila, E., Hovsepyan, R., Wilcox, G. \& Pessin, H. 2007, New data on the Late Prehistory of the Southern Caucasus. The excavations at Aratashen (Armenia). In: Les cultures du Caucase (VI-III millénaires). Leurs relations avec le Proche-Orient ancien (Lyonnet, B., Ed.), CNRS (Paléorient), Paris: p. 37-61. (en anglais) (« Nouvelles données sur la préhistoire récente au Caucase du sud. Les fouilles à Aratashen (Arménie) »)

Binder, D. 2007, PPN Pressure Technology: Views from Anatolia. In: Systèmes techniques et communautés du Néolithique précéramique (Astruc, L., Binder, D., Briois, F., Ed.), Éditions A.P.D.C.A., Antibes: p. 235-243. (en anglais) (« Technologie de la pression au PPN : du point de vue de l'Anatolie »)

Cauvin, J. 1989, La néolithisation du Levant et sa première diffusion. In: Néolithisations (Aurenche, O., Ed.), BAR International Series Vol. 516, British Archaeological Reports, Oxford: p. 3-36. (in French) (“Levant’s Neolithisation and its first diffusion”) 
Chabot, J. 2002, Tell 'Atij-Tell Gudeda. Industrie lithique. Analyse technologique et fonctionnelle. "Cahiers d’archéologie du CELAT » Vol. 13, et « Série Archéométrie » Vol. 3), Éditions du Centre interuniversitaire d'études sur les lettres, les arts et les traditions (CELAT), Québec, 225 p. (in French) (“Tell 'Atij and Tell Gudeda, Lithic Industry. Technological and Functional Analysis”)

Chabot, J. 2015, Technological and Functional Analysis of the Chipped Stone Artifacts Discovered at Tell Al-Raqa'i . In: Rural Archaeology in Early Urban Mesopotamia. Excavations at Tell Al-Raqai (Schwartz, G. Ed.), « Monumenta Archaeologica » Vol. 36, UCLA, Cotsen Institute of Archaeology Press, Los AngelesL p. 493-519. (en anglais) («Analyse technologique et fonctionnelle des artefacts en pierre taillée découverts à Tell Al-Raqai »)

Chabot, J., Dionne, M.-M., Duval, I. \& C. Gosselin, C. 2014, Décoder l’outil. Usure, utilisation et fonction de l'outillage lithique en préhistoire du Nord-Est. Archéologiques, 37: 48-68. (in French) ("To Decode the Tool: Use, Utilisation and Function of the lithic tools in North-East Prehistory”)

Chabot, J., Dionne, M.-M. \& Paquin, S. 2017, High magnification use-wear analysis of lithic artefacts from Northeastern America: Creation of an experimental database and integration of expedient tools. Quaternary International, special issue: New contributions to the functional analysis of prehistoric tools (Ollé, A., Pawlik, A., Longo, L., Skakun, N., Gibaja, J, \& Sala, R., Eds.), 427(B): 25-34. (en anglais) (« Analyse tracéologique à fort grossissement de matériel lithique provenant d’Amérique du nordest ») doi:10.1016/j.quaint.2015.11.061

Chabot, J. \& Eid, P. 2007, Stone tools from a Bronze Age Village (Tell Nusstell, Syria) in their wider context. Berythus Archaeological Studies, 50: 7-36. (en anglais) (« Outils en pierre taillée provenant d’un village de l'âge du bronze (Tell Nusstell, Syrie »)

Chabot, J. \& Eid, P. 2010, Tall Mozan: Le matériel lithique. In: Ausgrabungen 1998-2001 in der Zentralen Oberstadt von Tall Mozan/Urkeš: Bd. II: Die Kleinfunde (Bianchi, A. \& Wissing, A., Eds.), Eberhard-Karls-Universität, Tübingen: p. 803-820. (in French) ("Tall Mozan: the Lithic Material”)

Chabot, J., Gosselin, C., Eid, P. \& Varoutsikos, B. sous presse, Aknashen: Technotypological and functional analysis of the lithic assemblage. In: The Neolithic settlement of Aknashen,Armenia: the 2005-2009 and 2011-2015 excavation seasons (Chataigner, C., Ed.). Manuscript submitted and accepted, under revision. Archaeology Series, ArcheoPress, Oxford: 26 p. (en anglais) («Aknashen : analyses techno-typologique et fonctionnelle de l'assemblage lithique »)

Chabot, J. \& Pelegrin, J. 2012, Two Examples of Pressure Blade Production with a Lever:

Recent Research from Southern Caucasus (Armenia) and Northern Mesopotamia (Syria, Iraq), In: The Emergence of Pressure Blade Making. From Origins to Modern Experimentation (Desrosiers, P. Ed.), Springer, New York: p. 181-198. (en anglais) ( «Deux exemples de production par pression au levier : récentes recherches au Caucase (Arménie) et en Mésopotamie septentrionale (Syrie, Irak »)

Inizan, M.-L. 1991, Le debitage par pression : des choix culturels. In: 25 ans d'études technologiques en préhistoire. Bilan et perspectives. XIème Rencontres Internationales d'Archéologie et d'Histoire d'Antibes 18-20 octobre 1990. Association pour la Promotion et la Diffusion des Connaissances Archéologiques (A.P.D.C.A.), Antibes: p. 367-377. (in French) (“Pressure debitage: cultural choices”) 
Inizan, M.-L., Reduron, M., Roche, H \& Tixier, J. 1995, Technologie de la pierre taillée. Cercle de Recherches et d'Études Préhistoriques (CREP), Paris, 199 p. (in French) ("Knapped stone technology”)

Pelegrin, J. 1988, Débitage expérimental par pression: du plus petit au plus grand. In: Technologie préhistorique (Tixier, J., Ed.), Notes et monographies techniques Vol. 25, Centre National de la Recherche Scientifique (CNRS) Éditions, Paris: p. 37-53. (in French) ("Experimental Pressure Debitage”)

Pelegrin, J. 2002, Principes de la reconnaissance des méthodes et techniques de taille. In: Tell 'Atij-Tell Gudeda. Industrie lithique. Analyse technologique et fonctionnelle (Chabot. J., Ed.), CELAT, Québec: p. 215-226. (in French) ("Principles in order to identify knapping methods and techniques”)

Pelegrin, J. 2012a, New Experimental Observations for the Characterization of Pressure Blade Production Techniques, In: The Emergence of Pressure Blade Making. From Origins to Modern Experimentation (Desrosiers, P., Ed.), Springer, New York: p. 465-500. (en anglais) (« Nouvelles observations expérimentales pour identifier la production laminaire grâce aux techniques par pression »)

Pelegrin, J. 2012b, Grandes lames de l'Europe néolithique et alentour. In: L 'Europe, déjà, à la fin des temps préhistoriques (Marquet, J.-C. \& Verjux, C., Eds.), Actes de la table-ronde internationale, Revue Archéologique du Centre de la France - 38 Supplément, CNRS, Tours: p. 15-43. 2012b. (in French) ("Long Blades From European Neolithic and Surroundings”)

Tixier, J. 1984, Le débitage par pression. In: Préhistoire de la pierre taillée 2. Economie du débitage laminaire: technologie et expérimentation (IIIe table ronde de technologie lithique, Meudon-Bellevue, oct. 1982) (Tixier, J., Ed.), CREP, Paris: p. 57-70. (in French) ("Pressure Debitage”) 


\title{
Neolithic Industry of Long Obsidian Blades: the Case of Aknashen-Khatunarkh (Armenia, Early Sixth Millenium)
}

\author{
Jacques Chabot
}

Université Laval. Département des Sciences Historiques, 1030 avenue des Sciences-Humaines, Pavillon De

Koninck, Québec, Qc, G1V 0A6, Canada. Email: jacques.chabot@hst.ulaval.ca

\begin{abstract}
:
Aknashen (formerly called Khatunarkh) is a small Neolithic village of the Ararat valley located 25 kilometres away from Yerevan (capital of Armenia) and $5 \mathrm{~km}$ South-West of Echmiadzin (Vagharshapat). Aknashen is also located at only six kilometres of another Neolithic village which is contemporary: Aratashen, a site for which we recently published a technological study of the obsidian industry. Excavations have been taking place each year since 2004 at Aknashen. Concerning the lithic material, we identified two main chaînes opératoires on obsidian (which is an abundant raw material in this region): one concerns expedient tools made on flakes and the other one relates to long regular blades. This article will concentrate on this long blade industry obtained by three techniques: standing up pressure with a crutch, pressure with a lever and indirect percussion. Otherwise, the link between the application of these techniques and the complete chaîne opératoire of these blades is difficult to figure since elements are missing and we do not know for sure if, for example, the blades knapped by standing up pressure with a crutch were obtained after the progressive reduction of the cores knapped first using indirect percussion and then pressure with a lever. But even if we still don't understand various elements, such as the exact place (workshop) where knapping activities took place at the site, even if various "debitage" products have been found, at least this constitutes a proof that part of this chaîne opératoire was performed at the site. Furthermore, a systematic study of this material makes it possible to recognize the techniques used, but also to observe the great level of know-how of the specialists who carried out this work. Generally, standing up pressure with a crutch and indirect percussion, are two techniques used in order to obtain long regular blades, and these are better known (documented) than pressure with a lever, even if the latter has been identified in several cultures from the Neolithic, Chalcolithic and sometimes even during Early Bronze Age periods, nonetheless it has been the object of fewer studies of this kind. However, the identification of this high level of skill is important for many reasons, of course first from a pure technological point of view, but the presence of the technique of pressure with a lever on a given site can also constitute an excellent cultural marker in order to characterize cultures that are involved with it and also to help documenting more adequately exchanges (trade networks) that have been made and possible movements or contacts between populations (transmission of knowledge). In this article, a description of diagnostic specimens linked to each technique will be done and this material will be looked from a broader point of view (history of techniques and know-how) in order to have better knowledge of this culture and its possible origins (origin of this Neolithic still not precisely defined). As this research is still relatively new, many aspects are still to be confirmed and this work will carry on in the years to come.
\end{abstract}

Keywords: lithic technology; chaîne opératoire; technological know-how; Neolithic; obsidian; Caucasus; Northern Mesopotamia 